\title{
A new species of the Rana japonica group (Anura, Ranidae, Rana) from China, with a taxonomic proposal for the $R$. johnsi group
}

\author{
Han Wan ${ }^{\text {I, }}$, Zhi-Tong Lyu ${ }^{1,2,}$, Shuo Qi ${ }^{1,3}$, Jian Zhao ${ }^{4}$, Pi-Peng Li \\ Ying-Yong Wang'
}

I State Key Laboratory of Biocontrol/ The Museum of Biology, School of Life Sciences, Sun Yat-sen University, Guangzhou 510275, China 2 School of Ecology, Sun Yat-sen University, Guangzhou 510006, China 3 Institute of herpetology, Shenyang Normal University, Shenyang 110034, China 4 Shenzhen Shuanghuamu Biological Technology Co., Ltd., Shenzhen 51800, China

Corresponding author: Ying-Yong Wang (wangyy@mail.sysu.edu.cn); Pi-Peng Li (lipipeng@yahoo.com)

Academic editor: A. Crottini | Received 28 September 2019 | Accepted 6 May 2020 | Published 18 June 2020

http://zoobank.org/5184920F-C3AC-4133-B35F-6EODB6F77BDE

Citation: Wan H, Lyu Z-T, Qi S, Zhao J, Li P-P, Wang Y-Y (2020) A new species of the Rana japonica group (Anura, Ranidae, Rana) from China, with a taxonomic proposal for the $R$. johnsi group. ZooKeys 942: 141-158. https://doi. org/10.3897/zookeys.942.46928

\begin{abstract}
Rana jiulingensis sp. nov., a new species from Hunan and Jiangxi, southeastern China, is described. The new species is assigned to the $R$. japonica group. The clade comprising $R$. jiulingensis sp. nov. and $R$. dabieshanensis from Anhui is the sister taxon of $R$. omeimontis from Sichuan. Rana jiulingensis sp. nov. can be distinguished by the significant divergences in the $16 \mathrm{~S}$ and COI genes, and the combination of following morphological characters: body size medium, SVL 48.3-57.8 $\mathrm{mm}$ in adult males and 48.2-57.5 $\mathrm{mm}$ in adult females; dorsolateral fold straight; digits without circummarginal grooves; dorsal skin smooth; tibiotarsal articulation reaching forward beyond the tip of snout; heels overlapping; webbing formula of toes: I $1 \frac{1}{3}-2$ II $1 \frac{1}{3}-2^{1 / 3}$ III $1 \frac{1}{2}-2^{2} / 3$ IV $3-1 \frac{1}{3} \mathrm{~V}$; absence of vocal sacs in males; and presence of creamy white nuptial pad with tiny hoar spines on the finger I and reddish tubercles on loreal and temporal regions in breeding males. Furthermore, based on our results and the previous literature, $R$. zhengi is synonymized with $R$. sangzhiensis, and a new species group, the Rana johnsi group, is proposed for the $R$. johnsi and $R$. sangzhiensis. Currently, the subgenus Rana contains 41 recognized species, and the phylogenetic placements of several species remain unresolved.
\end{abstract}

* Contributed equally as the first authors.

Copyright Han Wan et al. This is an open access article distributed under the terms of the Creative Commons Attribution License (CC BY 4.0), which permits unrestricted use, distribution, and reproduction in any medium, provided the original author and source are credited. 


\section{Keywords}

morphology, phylogeny, Rana jiulingensis sp. nov., Rana sangzhiensis, Rana zhengi

\section{Introduction}

As the type genus of the family Ranidae Batsch, 1796, the concept of the true-frog genus Rana Linnaeus, 1758 has been discussed for a long time (Frost 2020). In a recent phylogenetic analysis (Yuan et al. 2016), Rana sensu lato was considered to be composed of nine clades, namely the subgenera Rana, Amerana Dubois, 1992, Liuhurana Fei, Ye, Jiang, Dubois \& Ohler, 2010, Aquarana Dubois, 1992, Lithobates Fitzinger, 1843, Zweifelia Dubois, 1992, Pantherana Dubois, 1992, Pseudorana Fei, Ye \& Huang, 1990, and an unnamed monotypic clade containing $R$. sylvatica (LeConte, 1825). However, this classification is still controversial, especially for the recognitions of the genera Lithobates and Pseudorana (Frost 2020). Nevertheless, the subgenus Rana, which is currently well recognized, contains 41 known species distributed from Europe to southeastern Asia. Among them, 23 species occur in China (AmphibiaWeb 2019). Recent researches on this subgenus have discovered new species from China and revised several taxonomic errors, indicating that the diversity and taxonomy of the subgenus Rana are still insufficiently understood (Yan et al. 2011; Zhou et al. 2015, 2017; Yuan et al. 2016; Wang et al. 2017; Yang et al. 2017; Zhao et al. 2017).

Based on morphological comparisons and geographical conditions, Fei et al. (2009) proposed three species groups for the Chinese species of the subgenus Rana: $R$. longicrus group, $R$. chensinensis group, and $R$. amurensis group. Subsequent phylogenetic analyses have revised several memberships of these groups (Yan et al. 2011; Zhou et al. 2015, 2017; Yuan et al. 2016; Wang et al. 2017; Zhao et al. 2017), and the nomenclature of

the $R$. longicrus group was replaced by the $R$. japonica group (Yang et al. 2017). Currently, 16 Chinese species are recognized as members of the three species groups. The $R$. japonica group contains nine species: $R$. $(R$.) chaochiaoensis Liu, 1946; $R$. $(R$.) chevronta $\mathrm{Hu} \& \mathrm{Ye}$, 1978; $R$. (R.) culaiensis $\mathrm{Li}, \mathrm{Lu} \& \mathrm{Li}, 2008 ; R$. $(R$.) dabieshanensis Wang, Qian, Zhang, Guo, Pan, Wu, Wang \& Zhang, 2017; R. (R.) hanluica Shen, Jiang \& Yang, 2007; R. (R.) jiemuxiensis Yan, Jiang, Chen, Fang, Jin, Li, Wang, Murphy, Che \& Zhang, 2011; $R$. $(R$.) longicrus Stejneger, 1898; $R$. (R.) omeimontis Ye \& Fei, 1993; and $R$. (R.) zhenhaiensis Ye, Fei \& Matsui, 1995. The $R$. chensinensis group contains four species: $R$. $(R$.) chensinensis David, 1875; $R$. (R.) dybowskii Günther, 1876; $R$. (R.) buanrenensis Liu, Zhang \& Liu, 1993; and $R$. (R.) kukunoris Nikolskii, 1918. The $R$. amurensis group has three species: $R$. $(R$.) amurensis Boulenger, 1886; $R$. (R.) coreana Okada, 1928; and $R$. (R.) luanchuanensis Zhao \& Yuan, 2017. However, species groups have not yet been proposed to accommodate the remaining seven species: $R$. $(R$.$) arvalis Nilsson, 1842 ; R$. $(R$.$) asiatica Bedriaga,$ 1898; $R$. (R.) maoershanensis Lu, Li \& Jiang, 2007; $R$. $(R$.) sauteri Boulenger, 1909; $R$. $(R$.$) johnsi Smith, 1921; R. (R.) sangzhiensis Shen, 1986; and R$. (R.) zhengi Zhao, 1999.

During herpetofaunal surveys in the Luoxiao Range, which is situated between the Jiangxi and Hunan provinces (Fig. 1), a series of Rana specimens was collected 


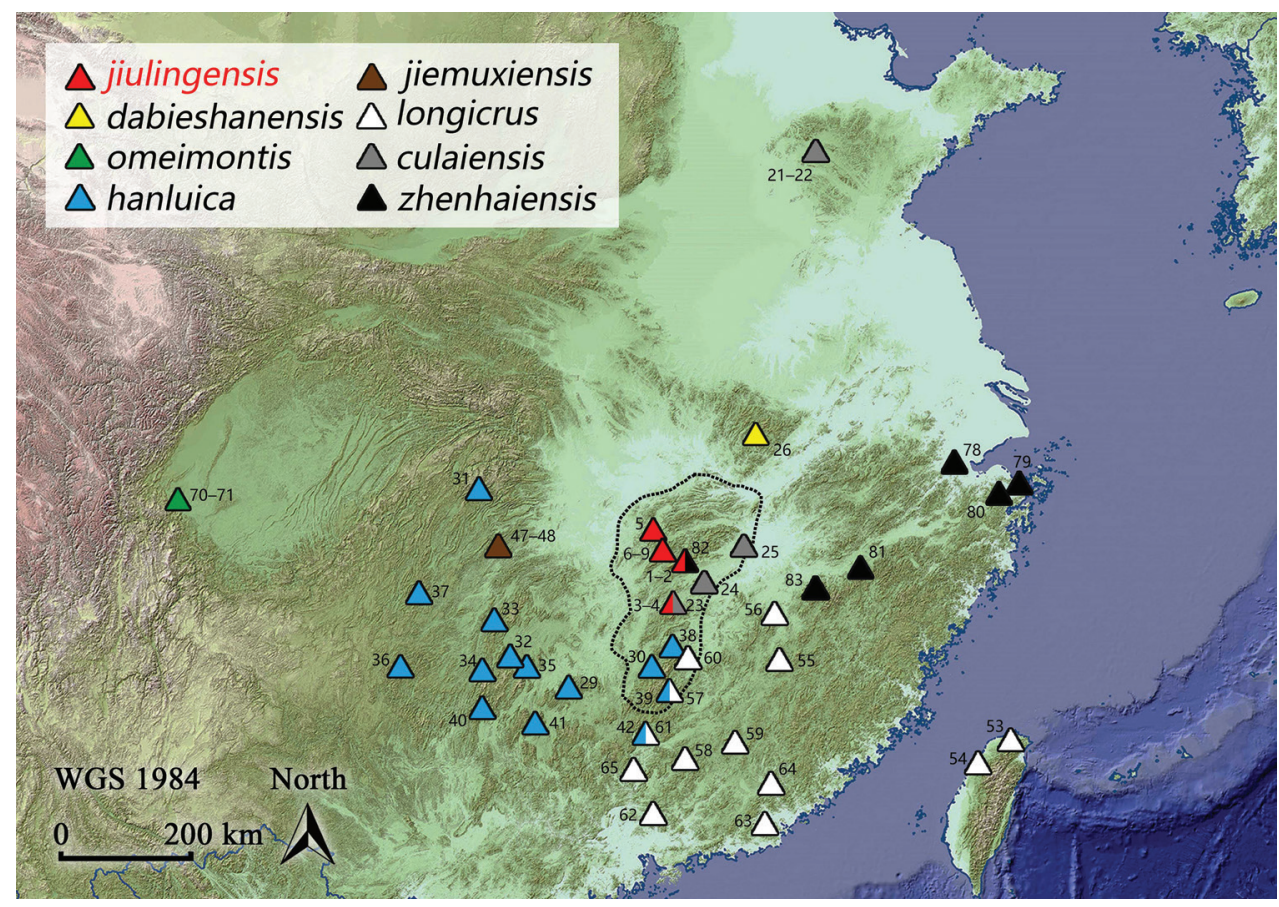

Figure I. Collecting localities of Rana samples used in this study. Dotted line shows the Luoxiao Range, where five Rana species are recorded. Numbers correspond to the ID in Table 1.

which can be assigned to the $R$. japonica group based on morphological characteristics. However, detailed examination of these specimens showed significant differences from all known congeners. Additional molecular analysis well supported the morphological identifications, demonstrating that these specimens formed an unnamed lineage within the $R$. japonica group. Therefore, we describe this series of specimens as a new species. Additionally, as revealed from our results and the previous literature, we suggest that $R$. zhengi should be synonymized with $R$. sangzhiensis, and we also propose a new species group, the Rana johnsi group, for the species $R$. johnsi and $R$. sangzhiensis.

\section{Materials and methods}

\section{Sampling and morphological analyses}

Eight unnamed specimens were collected from multiple localities of Jiangxi and Hunan provinces. All specimens were fixed in $10 \%$ buffered formalin, later transferred to 70\% ethanol, and deposited in the Museum of Biology, Sun Yat-sen University (SYS) and Chengdu Institute of Biology, Chinese Academy of Sciences (CIB), PR China. External measurements were made for the unnamed specimens with digital calipers (Neiko 01407A Stainless Steel 6-Inch Digital Caliper, USA) to the nearest $0.1 \mathrm{~mm}$. These measurements are as follows: 
SVL snout-vent length (from tip of snout to posterior margin of vent);

HL head length (from tip of snout to the articulation of the jaw);

HW head width (head width at the commissure of the jaws);

SL snout length (from tip of snout to the anterior corner of the eye);

IN internasal distance (distance between nares);

IO interorbital distance (minimum distance between upper eyelids);

ED eye diameter (from the anterior corner of the eye to posterior corner of the eye);

TD tympanum diameter (horizontal diameter of tympanum);

TED tympanum-eye distance (from anterior edge of tympanum to posterior corner of the eye);

HND hand length (from the proximal border of the outer palmar tubercle to the tip of digit III);

RAD radio-ulna length (from the flexed elbow to the proximal border of the outer palmar tubercle);

FTL foot length (from distal end of shank to the tip of digit IV);

TIB tibial length (from the outer surface of the flexed knee to the heel).

The morphological description follows the consistent definition by Fei et al. (2009). Sex and age were determined by examining the gonads. Webbing formula was based on Savage (1975). Comparison characters of known congeners were obtained from the literature (Stejneger 1898; Liu 1946; Liu et al. 1993; Ye et al. 1993, 1995; Lu et al. 2007; Shen et al. 2007; Li et al. 2008; Fei et al. 2009, 2012; Yan et al. 2011; Wang et al. 2017; Zhao et al. 2017) and 80 examined museum specimens listed in the Appendix 1.

\section{DNA Extraction, PCR amplification, and sequencing}

A total of 56 muscular samples of Rana were used, encompassing nine samples of the undescribed specimens, and 47 samples from 12 recognized species. All samples were attained from euthanasia specimens and then preserved in 95\% ethanol and stored at $-40{ }^{\circ} \mathrm{C}$. Genomic DNA were extracted from muscle tissue samples, using DNA extraction kit from Tiangen Biotech (Beijing) Co., Ltd. Two mitochondrion genes, namely partial $16 \mathrm{~S}$ ribosomal RNA gene (16S) and partial cytochrome c oxidase 1 gene (COI), were amplified. Primers used for 16S were L3975 (5'-CGCCTGTTTACCAAAAACAT-3') and H4551 (5'-CCGGTCTGAACTCAGATCACGT-3') following Simon et al. (1994), and L2A (5'-CCAAACGAGCCTAGTGATAGCTGGTT-3') and H10 (5'-TGATTACGCTACCTTTGCACGGT-3') following Chen et al. (2013), for COI were dgLCO (5'-GGTCAACAAATCATAAAGAYATYGG-3') and dgHCO (5'-AAACTTCAGGGTGACCAAARAAYCA-3') following Meyer et al. (2005). PCR amplifications were processed with the cycling conditions that initial denaturing step at $95^{\circ} \mathrm{C}$ for $4 \mathrm{~min}, 35$ cycles of denaturing at $94^{\circ} \mathrm{C}$ for $40 \mathrm{~s}$, annealing at $53{ }^{\circ} \mathrm{C}$ (for 16S) $/ 48^{\circ} \mathrm{C}$ (for COI) for $40 \mathrm{~s}$ and extending at $72{ }^{\circ} \mathrm{C}$ for $60 \mathrm{~s}$, and a final extending step at $72{ }^{\circ} \mathrm{C}$ for $10 \mathrm{~min}$. PCR products were purified with spin columns and then 
Table I. Localities, voucher information, and GenBank numbers for all samples of the genus Rana used in this study $\left({ }^{*}=\right.$ type localities).

\begin{tabular}{|c|c|c|c|c|c|}
\hline ID & Species & Localities & Voucher no. & $16 S$ & COI \\
\hline 1 & R. jiulingensis & China: Jiangxi: Mt Guanshan * & SYS a005519 & MT408985 & MT418647 \\
\hline 2 & R. jiulingensis & China: Jiangxi: Mt Guanshan * & SYS a006999 & MT408994 & MT418656 \\
\hline 3 & R. jiulingensis & China: Jiangxi: Mt Wugong & SYS a002584 & MT408964 & MT418626 \\
\hline 4 & R. jiulingensis & China: Jiangxi: Mt Wugong & SYS a002585 & MT408965 & MT418627 \\
\hline 5 & R. jiulingensis & China: Hunan: Mt Mufu & SYS a005511 & MT408984 & MT418646 \\
\hline 6 & R. jiulingensis & China: Hunan: Mt Dawei & SYS a006451 & MT408989 & MT418651 \\
\hline 7 & R. jiulingensis & China: Hunan: Mt Dawei & SYS a006494 & MT408990 & MT418652 \\
\hline 8 & R. jiulingensis & China: Hunan: Mt Dawei & SYS a006495 & MT408991 & MT418653 \\
\hline 9 & R. jiulingensis & China: Hunan: Mt Dawei & SYS a006496 & MT408992 & MT418654 \\
\hline 10 & R. amurensis & China: Heilongjiang: Taiyang Island & SYNU 11100267 & KF020589 & KF020603 \\
\hline 11 & R. amurensis & China: Liaoning, Zhangwu & SYNU 11100268 & KU343216 & KU343216 \\
\hline 12 & R. arvalis & Germany: Lower Saxony & No voucher & AY147938 & I \\
\hline 13 & R. asiatica & China: Xinjiang: 47tuan & KIZ XJ0251 & KX269200 & I \\
\hline 14 & R. chaochiaoensis & China: Sichuan: Zhaojue * & SYS a001815 & MT409007 & MT418669 \\
\hline 15 & R. chaochiaoensis & China: Sichuan: Zhaojue * & SYS a001816 & MT408957 & MT418619 \\
\hline 16 & R. chensinensis & China: Shaanxi: Huxian * & KIZ RD05SHX01 & KX269186 & JF939080 \\
\hline 17 & R. chensinensis & China: Henan: Mt Yawu & SYS a002392 & MT408962 & MT418624 \\
\hline 18 & R. chensinensis & China: Henan: Mt Yawu & SYS a002393 & MT408963 & MT418625 \\
\hline 19 & R. coreana & South Korea & MMS 223 & KX269202 & MF149928 \\
\hline 20 & R. coreana & China: Shandong: Mt Kunyu & SYNU 08090641 & MT409004 & MT418666 \\
\hline 21 & R. culaiensis & China: Shandong: Mt Culai * & KIZ SD080501 & KX269190 & JF939082 \\
\hline 22 & R. culaiensis & China: Shandong: Mt Culai * & SYNU 08090549 & MT409006 & MT418668 \\
\hline 23 & R. culaiensis & China: Jiangxi: Mt Wugong & SYS a002634 & MT408966 & MT418628 \\
\hline 24 & R. culaiensis & China: Jiangxi: Shanggao & SYS a002641 & MT408967 & MT418629 \\
\hline 25 & R. culaiensis & China: Jiangxi: Mt Meiling & SYS a004239 & MT408971 & MT418633 \\
\hline 26 & R. dabieshanensis & China: Anhui: Dabie Mountains area * & AHU 2016R001 & MF172963 & l \\
\hline 27 & R. dybowskii & Russia: Primorye: Khasanskii & MSUZP-IVM-1d & KX269188 & I \\
\hline 28 & R. dybowskii & China: Jilin: Mt Laoling & SYNU 11070163 & MT409005 & MT418667 \\
\hline 29 & R. hanluica & China: Hunan: Mt Yangming * & SYS a001137 & MT408956 & MT418618 \\
\hline 30 & R. hanluica & China: Hunan: Mt Bamian & SYS a004086 & MT408969 & MT418631 \\
\hline 31 & R. hanluica & China: Hunan: Mt Badagong & SYS a004298 & MT408973 & MT418635 \\
\hline 32 & R. hanluica & China: Hunan: Mt Yunshan & SYS a004359 & MT408977 & MT418639 \\
\hline 33 & R. hanluica & China: Hunan: Mt Xuefeng & SYS a007216 & MT408999 & MT418661 \\
\hline 34 & R. hanluica & China: Hunan: Suining & SYS a007250 & MT409000 & MT418662 \\
\hline 35 & R. hanluica & China: Hunan: Mt Shunhuang & SYS a007259 & MT409001 & MT418663 \\
\hline 36 & R. hanluica & China: Guizhou: Mt Leigong & SYS a002233 & MT408959 & MT418621 \\
\hline 37 & R. hanluica & China: Guizhou: Mt Fanjing & SYS a004346 & MT408976 & MT418638 \\
\hline 38 & R. hanluica & China: Jiangxi: Mt Jinggang & SYS a004033 & MT408968 & MT418630 \\
\hline 39 & R. hanluica & China: Jiangxi: Mt Qiyun & SYS a004087 & MT408970 & MT418632 \\
\hline 40 & R. hanluica & China: Guangxi: Longsheng & SYS a002286 & MT408960 & MT418622 \\
\hline 41 & R. hanluica & China: Guangxi: Mt Dupangling & SYS a005087 & MT408980 & MT418642 \\
\hline 42 & R. hanluica & China: Guangdong: Renhua & SYS a007100 & MT408998 & MT418660 \\
\hline 43 & R. huanrenensis & China: Liaoning: Huanren * & SYNU 07040035 & KF204642 & KX139725 \\
\hline 44 & R. huanrenensis & China: Liaoning: Huanren * & $\mathrm{y}-\mathrm{d} 20130058$ & KT588071 & KT588071 \\
\hline 45 & R. japonica & Japan: Isumi-shi: Chiba Prefecture & KIZ YPX11775 & KX269220 & JF939101 \\
\hline 46 & R. japonica & Japan: Isumi-shi: Chiba Prefecture & NNRj & AB728192 & 1 \\
\hline 47 & R. jiemuxiensis & China: Hunan: Jiemuxi* & SYS a004318 & MT408975 & MT418637 \\
\hline 48 & R. jiemuxiensis & China: Hunan: Jiemuxi * & SYS a004319 & MT409008 & MT418670 \\
\hline 49 & R. johnsi & Vietnam: Lam Dong: Loc Bao & ABV 00203 & KX269182 & I \\
\hline 50 & R. kukunoris & China: Qinghai: Qinghai Lake* & KIZ CJ06102001 & KX269185 & JF939073 \\
\hline
\end{tabular}




\begin{tabular}{|c|c|c|c|c|c|}
\hline ID & Species & Localities & Voucher no. & $16 S$ & COI \\
\hline 51 & R. kukunoris & China: Sichuan: Hongyuan & SYS a006652 & MT409009 & MT418671 \\
\hline 52 & R. kukunoris & China: Sichuan: Hongyuan & SYS a006653 & MT408993 & MT418655 \\
\hline 53 & R. longicrus & China: Taiwan:Taipei * & Not given & AB058881 & 1 \\
\hline 54 & R. longicrus & China: Taiwan: Miaoli: Xiangtianhu & NMNS 15022 & KX269189 & I \\
\hline 55 & R. longicrus & China: Fujian: Mt Yashu & SYS a005905 & MT408987 & MT418649 \\
\hline 56 & R. longicrus & China: Jiangxi: Mt Magu & SYS a007038 & MT408996 & MT418658 \\
\hline 57 & R. longicrus & China: Jiangxi: Mt Qiyun & SYS a002355 & MT408961 & MT418623 \\
\hline 58 & R. longicrus & China: Jiangxi: Mt Jiulian & SYS a004487 & MT408978 & MT418640 \\
\hline 59 & R. longicrus & China: Jiangxi: Mt Sanbai & SYS a005892 & MT408986 & MT418648 \\
\hline 60 & R. longicrus & China: Jiangxi: Suichuan & SYS a007097 & MT408997 & MT418659 \\
\hline 61 & R. longicrus & China: Guangdong: Renhua & SYS a000735 & MT408954 & MT418616 \\
\hline 62 & R. longicrus & China: Guangdong: Mt Nankun & SYS a000754 & MT408955 & MT418617 \\
\hline 63 & R. longicrus & China: Guangdong: Pu’ning & SYS a004605 & MT408979 & MT418641 \\
\hline 64 & R. longicrus & China: Guangdong: Mt Tonggu & SYS a005218 & MT408981 & MT418643 \\
\hline 65 & R. longicrus & China: Guangdong: Yingde & SYS a007519 & MT409003 & MT418665 \\
\hline 66 & R. maoershanensis & China: Guangxi: Mt Maoershan * & SYNU 08030061 & HQ228162 & I \\
\hline 67 & R. maoershanensis & China: Guangxi: Mt Maoershan * & SYNU 08030062 & HQ228163 & I \\
\hline 68 & R. luanchuanensis & China: Henan: Luanchuan * & KIZ 047452 & l & MF149923 \\
\hline 69 & R. luanchuanensis & China: Henan: Luanchuan * & KIZ 047393 & I & MF149924 \\
\hline 70 & R. omeimontis & China: Sichuan: Mt Emei * & SYS a005304 & MT408982 & MT418644 \\
\hline 71 & R. omeimontis & China: Sichuan: Mt Emei * & SYS a005305 & MT408983 & MT418645 \\
\hline 72 & R. sangzhiensis & China: Hunan: Mt Tianping * & SYS a004286 & MT408972 & MT418634 \\
\hline 73 & R. sangzhiensis & China: Hunan: Mt Tianping * & SYS a004299 & MT408974 & MT418636 \\
\hline 74 & R. zhengi & China: Sichuan: Hongya: Zhangcun * & $\begin{array}{c}\text { SCUM } \\
0405190 \mathrm{CJ}\end{array}$ & KX269206 & MF149929 \\
\hline 75 & R. zhengi & China: Sichuan: Hongya: Zhangcun * & KIZ YP06057 & DQ289104 & l \\
\hline 76 & R. sauteri & China: Taiwan: Kaohsiung * & $\begin{array}{c}\text { SCUM } \\
0405175 \mathrm{CJ}\end{array}$ & KX269204 & / \\
\hline 77 & R. shuchinae & China: Sichuan: Zhaojue & CIB HUI040009 & KX269210 & l \\
\hline 78 & R. zhenhaiensis & China: Zhejiang: Hangzhou & SYNU 08040100 & KF020599 & KF020613 \\
\hline 79 & R. zhenhaiensis & China: Zhejiang: Zhenhai * & KIZ 0803271 & KX269218 & JF939065 \\
\hline 80 & R. zhenhaiensis & China: Zhejiang: Fenghua & SYS a006208 & MT408988 & MT418650 \\
\hline 81 & R. zhenhaiensis & China: Jiangxi: Mt Tongbo & SYS a001952 & MT408958 & MT418620 \\
\hline 82 & R. zhenhaiensis & China: Jiangxi: Mt Guanshan & SYS a007000 & MT408995 & MT418657 \\
\hline 83 & R. zhenhaiensis & China: Jiangxi: Mt Yangjifeng & SYS a007422 & MT409002 & MT418664 \\
\hline
\end{tabular}

sequenced with both forward and reverse primers using BigDye Terminator Cycle Sequencing Kit per the guidelines, on an ABI Prism 3730 automated DNA sequencer by Shanghai Majorbio Bio-pharm Technology Co., Ltd. All sequences were deposited in GenBank (Table 1).

\section{Phylogenetic analyses}

For phylogenetic analyses, 26 additional sequences from all known Chinese congeners of the subgenus Rana (except $R$. (R.) chevronta) and an out-group sequence of $R$. (Liuburana) shuchinae Liu, 1950 were obtained from GenBank and incorporated into our dataset. Detailed information of these materials is shown in Table 1 and Figure 1. DNA sequences were aligned respectively by the Clustal W algorithm with default 
parameters (Thompson et al. 1997). For GenBank sequences that lack information for part of the segments, we filled the blank sites with "N". The aligned data was trimmed for allowing no gap positions and default parameters in Gblocks version 0.91b (Castresana 2000). All newly obtained sequences were deposited in GenBank (Table 1). PartitionFinder 2 was used to test the best partitioning scheme and jModelTest v2.1.2 was used to test the best fitting nucleotide substitution models, resulting in the best fit models for the partitions of COI and $16 S$ as GTR + I + G. Sequenced data were analyzed using Bayesian inference (BI) in MrBayes 3.2.4 (Ronquist et al. 2012), and maximum likelihood (ML) in RaxmlGUI 1.3 (Silvestro and Michalak 2012). Two independent runs were conducted in a BI analysis, each of which was performed for 10,000,000 generations and sampled every 1000 generations with the first $25 \%$ samples discarded as burn-in, resulting in a potential scale reduction factor (PSRF) of $<0.005$. In ML analysis, the bootstrap consensus tree inferred from 1000 replicates was used to represent the evolutionary history of the taxa analyzed. Pairwise distances were respectively calculated b in MEGA 6 using the uncorrected $p$-distance model.

\section{Results}

\section{Morphological comparison}

The unnamed specimens from Jiangxi and Hunan are assigned to the Rana japonica group based on the following combined characteristics: digits without circummarginal grooves, and dorsolateral fold distinct, extending straight from the posterior margin of the upper eyelid to above the groin. Therefore, we compare the new species with the species of the $R$. japonica group.

The new species differs from Rana dabieshanensis in the following characters: head length significantly larger than head width, HW/HL 0.82 in males and 0.85 in females (vs almost equal); supratympanic fold absent (vs distinct); tympanum diameter significantly smaller than eye diameter with $\mathrm{TD} / \mathrm{ED}=0.63-0.87$ (vs equal); relative toe lengths I $<$ II $<$ III $<$ V $<$ IV (vs I $<$ II $<$ V $<$ III $<$ IV); toe webbing formula I 11/3 - 2 II $1 \frac{1}{3}-2^{1 / 3}$ III $1 \frac{1}{2}-2^{2 / 3}$ IV $3-1 \frac{1}{3} \mathrm{~V}$ (vs I $2-1$ II $2^{+}-1^{+}$III $3-2$ IV $2-2^{+} \mathrm{V}$ ); and nuptial pad creamy white in breeding males (vs gray-blackish).

The new species differs from $R$. omeimontis as follows: body size smaller, $S V L=$ 48.2-57.5 $\mathrm{mm}$ in adult females (vs 61.7-70.3 $\mathrm{mm}$ in females); head length significantly larger than head width, HW/HL $=0.82$ in males and 0.85 in females (vs head length slightly larger than head width, $\mathrm{HW} / \mathrm{HL}=0.94$ in males and 0.92 in females); and supernumerary tubercles present below the bases of each finger (vs absent).

The new species further differs from $R$. hanluica as follows: supratympanic fold absent (vs present); toe webbing formula I $1 \frac{1}{3}-2$ II $1 \frac{1}{3}-21 / 3$ III $1 \frac{1}{2}-22 / 3$ IV $3-1 \frac{1}{3} \mathrm{~V}$ (vs I $1 \frac{1}{3}-1 \frac{1}{3}$ II $1-2$ III $1 \frac{1}{3}-2 \frac{1}{2}$ IV $2^{1 / 3}-1 \mathrm{~V}$ ); reddish tubercles present on loreal and temporal regions in breeding males (vs absent, but white horny spines present around loreal and temporal regions, upper eyelids, and snout in breeding males). The new 
species differs from $R$. longicrus in having: internarial distances larger than interorbital distances (vs smaller) and toe webbing formula I $1 \frac{1}{3}-2$ II $1 \frac{1}{3}-2^{1 / 3}$ III $1 \frac{1}{2}-22 / 3$ IV $3-1 \frac{1}{3} \mathrm{~V}$ (vs I $1 \frac{2}{3}-21 / 3$ II $1 \frac{1}{2}-22 / 3$ III $12 \frac{1}{3}-31 / 2$ IV $31 \frac{1}{3}-1 \frac{1}{2} \mathrm{~V}$ ); from $R$. zhenhaiensis: supratympanic fold absent (vs present), dorsolateral fold extending straight from posterior margin of upper eyelid to above groin (vs dorsolateral fold slightly curved above tympanum), two outer metacarpal tubercles distinctly separated (vs merged at base), tibio-tarsal articulation reaching forward beyond tip of snout (vs around nostril), and nuptial pad creamy white in breeding males (vs gray or gray-brownish); from $R$. culaiensis: dorsolateral fold extending straight from posterior margin of upper eyelid to above groin (vs dorsolateral fold slightly curved above tympanum), and tibio-tarsal articulation reaching forward beyond tip of snout (vs at nostril); from $R$. jiemuxiensis: dorsolateral fold extending straight from posterior margin of upper eyelid to above groin (vs dorsolateral fold slightly curved above tympanum), head length significantly larger than head width (vs slightly larger), internarial distances larger than interorbital distances (vs smaller), and two outer metacarpal tubercles distinctly separated (vs merged at base); from $R$. chaochiaoensis: supratympanic fold absent (vs present), internarial distances larger than interorbital distances (vs smaller), and toe webbing formula I $1 \frac{1}{3}-2$ II $1 \frac{1}{3}-2 \frac{1}{3}$ III $1 \frac{1}{2}-2 \frac{2}{3}$ IV $3-1 \frac{1}{3}$ V (vs I $1-12 \frac{1}{3}$ II $1 \frac{1}{3}-2$ III $1 \frac{1}{2}-2 \frac{1}{2}$ IV $22 / 3-1 \mathrm{~V}$ ); from $R$. japonica: outer metacarpal tubercles present (vs absent), tibio-tarsal articulation reaching forward beyond tip of snout (vs reaching or beyond tip of snout in males, reaching at center of eye or beyond nostril in females), nuptial pad creamy white and divided into three parts (vs nuptial pads grayish brown or yellowish brown and divided into two parts).

From Rana chevronta, which lacks molecular data, the new species can be distinguished by its larger body size, SVL $=48.3-57.8 \mathrm{~mm}$ in adult males (vs 39.7$44.0 \mathrm{~mm}$ ), head length significantly larger than head width (vs almost equal), relative finger lengths I $<$ II $<$ IV $<$ III (vs II $<$ IV $<$ I $<$ III), and nuptial pad creamy white and divided into three parts in breeding males (vs purplish gray and undivided).

\section{Phylogenetic analyses}

The ML and BI analyses resulted in essentially identical topologies and are integrated in Figure 2, in which the major nodes are sufficiently supported with the Bayesian posterior probabilities $(\mathrm{BPP})>0.95$ and the bootstrap supports (BS) for maximum likelihood analysis $>85$. The pairwise distances based on COI and 16S genes among all samples are given in the Supplementary material, Tables S1 and S2, respectively.

The Rana samples representing the new species are grouped in a distinct and robust monophyletic lineage with high support $(\mathrm{BPP}=1.00$ and $\mathrm{BS}=100)$ and low divergence (mean $0.3 \%$, ranging $0.0-0.6 \%$ in COI, and mean $0.1 \%$, ranging $0.0-0.5 \%$ in $16 S$ ); they form a separate evolutionary lineage within the $R$. japonica group. This lineage from Jiangxi and Hunan is close to $R$. dabieshanensis from Anhui and $R$. omeimontis from Sichuan. The smallest genetic distance between this lineage and a previously recognized species is $3.4-4.0 \%$ in COI (with $R$. omeimontis) and $1.6-2.0 \%$ in $16 \mathrm{~S}$ 
BS/BPP

* : $\mathrm{BS}=100$ or $\mathrm{BPP}=1.00$

0.02

(R) subgenus Rana

(L) subgenus Liuhurana

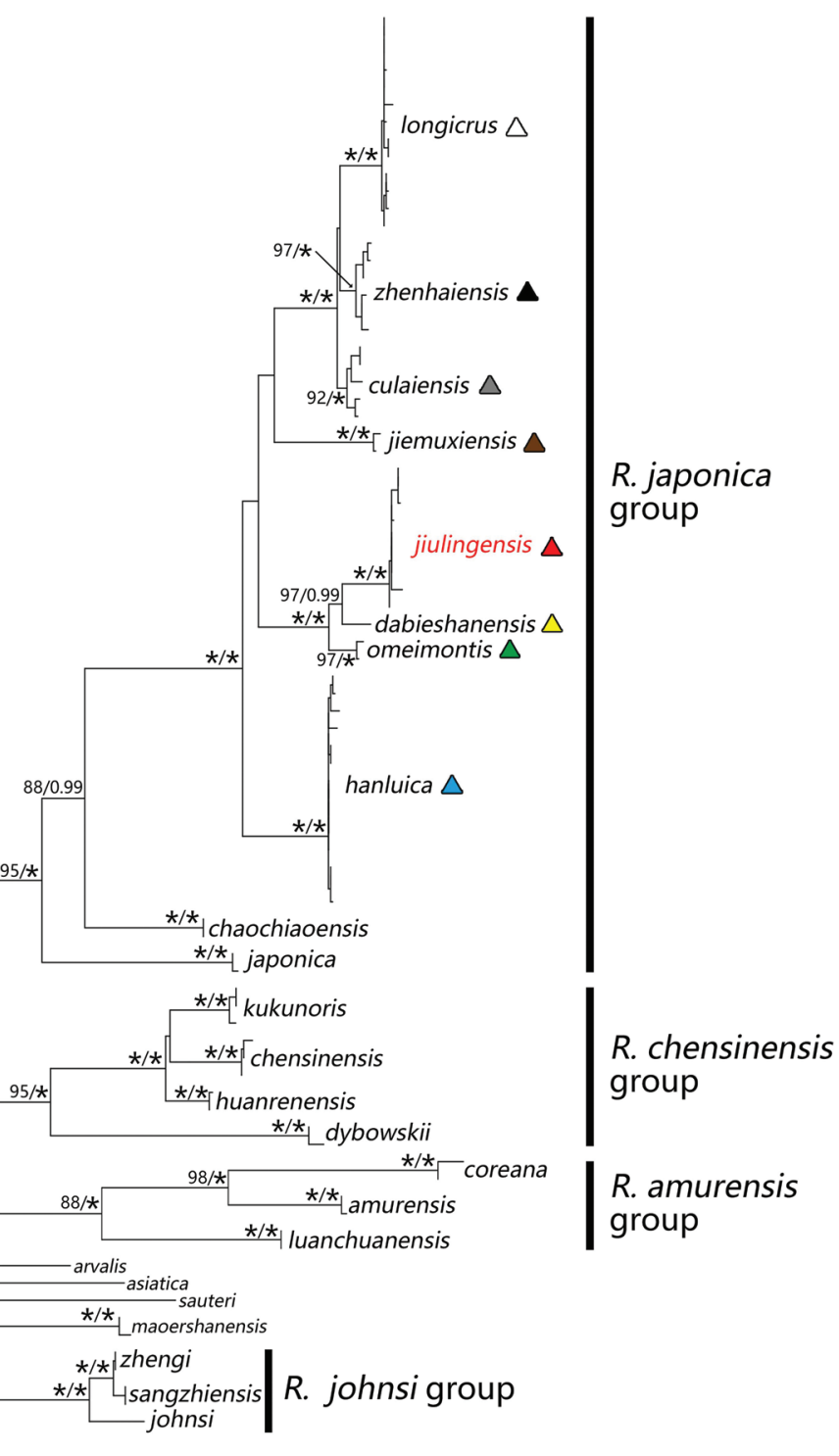

Figure 2. Bayesian inference and maximum-likelihood phylogenies based on mitochondrial 165 and COI genes.

(with $R$. dabieshanensis), which are significant when compared to all other recognized species (e.g. 2.8-3.6\% in COI between $R$. longicrus and $R$. culaiensis; $1.2-1.3 \%$ in $16 \mathrm{~S}$ between $R$. dabieshanensis and $R$. omeimontis).

Therefore, based on the significant morphological differences and phylogenetic divergence, these specimens from Jiangxi and Hunan represent a distinct evolutionary lineage and are described as a new species, Rana jiulingensis sp. nov. 


\section{Taxonomic account}

\section{Rana (Rana) jiulingensis Wan, Lyu \& Wang, sp. nov.}

http://zoobank.org/2E012E54-EFA3-4AA3-9B9F-0F884305AABD

Holotype. SYS a005519 (Fig. 3), adult male, collected by Zhi-Tong Lyu, Jian Wang and Hai-Long He on 14 September 2016 from Guanshan Nature Reserve (28.5535N, 114.5878E; ca 300 m a.s.l.), Yifeng County, Jiangxi province, PR China.

Paratypes. Seven adult specimens. Females SYS a002584-2585 collected by Jian Zhao on 8 May 2014 from Mt Wugong (27.4607N, 114.2059E; ca 1100 m a.s.l.), Anfu
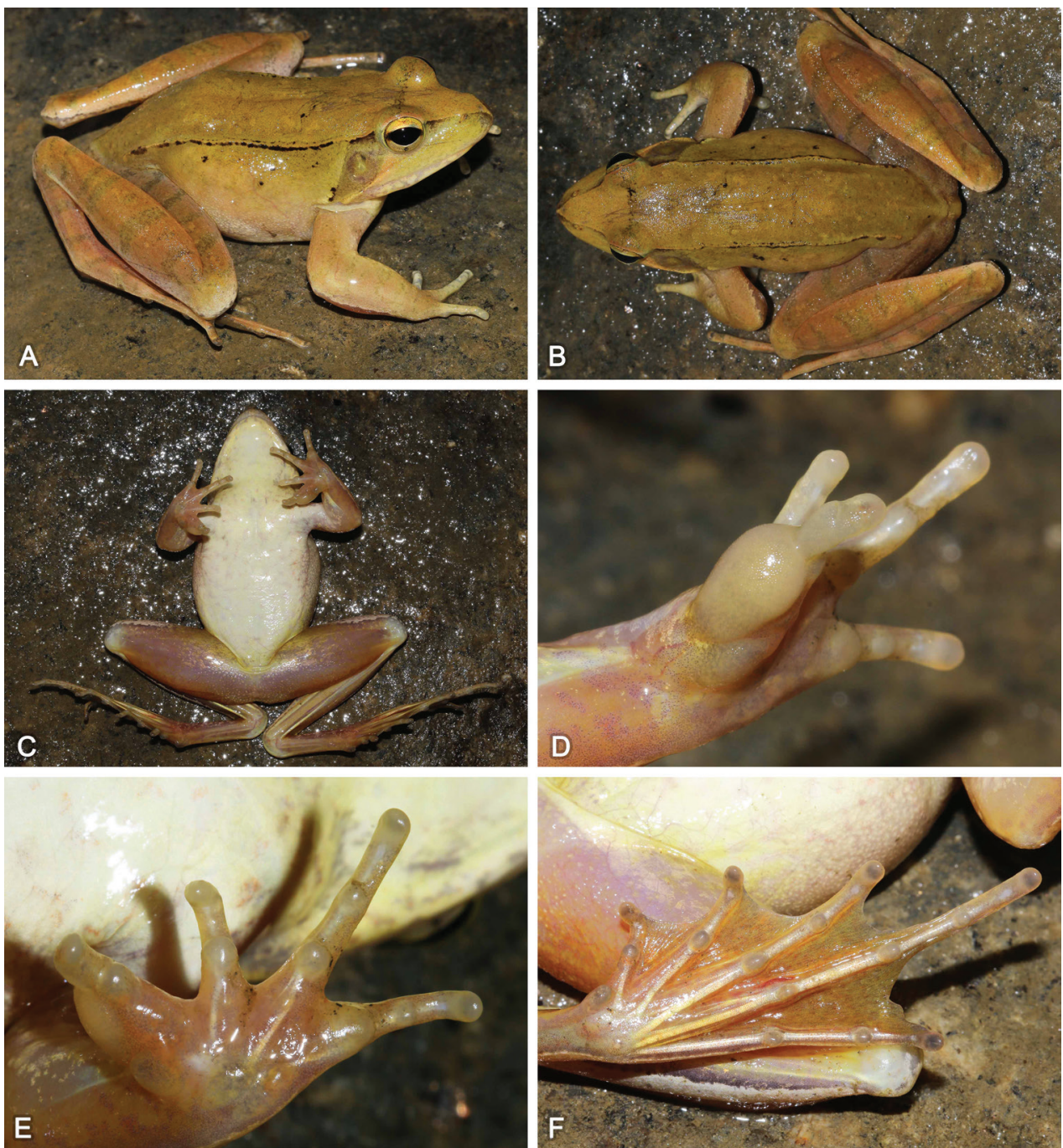

Figure 3. Morphological features of the adult male holotype SYS a005519 of Rana jiulingensis sp. nov. in life. A dorsolateral view $\mathbf{B}$ dorsal view $\mathbf{C}$ ventral view $\mathbf{D}$ grey nuptial pad $\mathbf{E}$ left hand $\mathbf{F}$ left foot. 
County, Jiangxi province. Male SYS a005511 collected by Zhi-Tong Lyu, Jian Wang and Hai-Long He on 13 September 2016 from Mt Mufu (28.9750N, 113.8304E; ca $1200 \mathrm{~m}$ a.s.l.), Pingjiang County, Hunan province. Males SYS a006494, SYS a006495/ CIB 110014, and females SYS a006451, 6496, collected by Zhi-Tong Lyu on 5-6 August 2017 from Mt Dawei (28.4250N, 114.0805E; ca 800 m a.s.l.), Liuyang City, Hunan province.

Etymology. The specific name jiulingensis is in reference to the type locality, Guanshan Nature Reserve in Jiuling Mountains.

Suggested common name. Jiuling Mountains Brown Frog (in English), Jiu Ling Shan Lin Wa (九岭山林蛙 in Chinese)

Diagnosis. Rana jiulingensis sp. nov. is distinguished by the following morphological characteristics: (1) body medium-sized, SVL $=48.3-57.8(51.7 \pm 4.3, n=4) \mathrm{mm}$ in adult males, 48.2-57.5 (50.8 $\pm 4.4, n=4) \mathrm{mm}$ in adult females; (2) head length significantly larger than head width; (3) supratympanic fold absent; (4) dorsolateral fold distinct and thin, extending straight from posterior margin of upper eyelid to above groin; (5) internarial distances larger than interorbital distances; (6) tympanum diameter significantly smaller than eye diameter, $\mathrm{TD} / \mathrm{ED}=0.63-0.87$; (7) fingers without circummarginal grooves, unwebbed, relative finger lengths I $<$ II $<$ IV $<$ III; (8) presence of supernumerary tubercles below the bases of each finger, presence of three separated metacarpal tubercles; (9) toes without circummarginal grooves, toe webbing formula: I $1 \frac{1 / 3}{3}-2$ II $1 \frac{1}{3}-2^{1 / 3}$ III $1 \frac{1}{2}-2^{2} / 3$ IV $3-1 \frac{1}{3} \mathrm{~V}$, relative toe lengths I $<$ II $<$ III $<\mathrm{V}<\mathrm{IV}$; (10) tibio-tarsal articulation reaching forward beyond tip of snout; (11) heels overlapping; (12) dorsal skin smooth, flanks smooth with few granules; (13) absence of vocal sacs in males; (14) breeding males possess creamy white nuptial pad with tiny hoar spines on the finger I, divided into three parts; (15) presence of reddish tubercles on loreal and temporal regions in breeding males.

Description of holotype. SYS a005519, adult male, SVL $57.8 \mathrm{~mm}$. Head length significantly larger than head width $(\mathrm{HW} / \mathrm{HL}=0.85)$; snout pointed and projecting; nostril closer to tip of snout than eye; canthus rostralis distinct; internasal distance slightly larger than interorbital distance; tympanum rounded, smaller than eye (TD/ $\mathrm{ED}=0.72$ ); tympanic rim prominent; pupil horizontal; loreal region concave, sloping outwards; vomerine teeth present; tongue deeply notched posteriorly; vocal sacs absent.

Forearms 0.19 of SVL and hand 0.26 of SVL; fingers slender, without web but with narrow fringe; tip of fingers rounded, not expanded, without circummarginal grooves; relative finger lengths I < II < IV < III; subarticular tubercles significantly prominent, rounded; distinct, small, rounded supernumerary tubercles below the bases of each finger; inner metacarpal tubercle indistinct, ovoid, partly covered by nuptial pad; two outer metacarpal tubercles distinctly separated, slightly larger, long elliptic; nuptial pad with tiny spines on the finger I, divided into three parts, the basal one around the inner metacarpal tubercle and partly covering it, the largest one from the edge of the basal one to the subarticular tubercle of finger I, the smallest one extending from the edge of the biggest one to the tip of finger I.

Tibia 0.63 of SVL and foot 0.88 of SVL; heels overlapping when hindlimbs flexed at right angles to axis of body; tibio-tarsal articulation reaching forward beyond the tip 
of snout when hindlimb stretched along the side of the body; relative toe lengths I $<$ II $<$ III $<$ V $<$ IV; toes webbing formula: I 11/3 - 2 II 11/3 - 21/3 III $1 \frac{1 / 2}{2}-2 \frac{2}{3}$ IV $3-1 \frac{1}{3}$ V; absence of lateral fringes on the lateral edges of toes I and V; subarticular tubercles oval and distinct; inner metatarsal tubercle large, ovoid, outer metatarsal tubercle small.

Dorsal skin smooth with sparse tiny granules; several small tubercles on flank; supratympanic fold absent; dorsolateral fold distinct and thin, extending straight from posterior margin of upper eyelid to above groin; several tiny granules on the skin of loreal and temporal regions; ventral surface smooth, large flattened tubercles densely arranged on the rear of thigh and around vent.

Coloration of holotype. In life, dorsal surface yellowish brown with few black spots; black speckles forming a linear stripe between eyelids; dorsolateral fold intermittently edged with black on two sides; loreal region yellowish; temporal region yellowish, slightly tinged with grey; tiny granules on loreal and temporal regions reddish; dorsal forelimbs and hindlimbs reddish with indistinct greenish grey transverse bars. Throat yellowish; chest and belly creamy white; ventral surface of forelimbs and hindlimbs flesh color; nuptial pad creamy white; tubercles around vent yellowish.

In preservative, dorsal surface turns grey with black spots and light grey patches; limbs taupe with brown transverse bars. Ventral surface white, with greyish mottling on throat and belly; ventral surface of limbs beige; hands and toe webs dark grey.

Variations. Measurements of type series specimens are given in Table 2. Coloration of dorsal skin varies from brown to yellowish brown (Fig. 4). Black edges on dorsolateral fold indistinct in all paratypes. SYS a006495 and 6496 with V-shaped mark. The number of transverse bars ranges from two to five on forearms, three or four on thigh, and three to six on tibia.

Distribution and ecology. Currently, Rana jiulingensis sp. nov. is known from Guanshan Nature Reserve in the Jiuling Mountains and Mount Wugong in the Wugong Mountains of northwestern Jiangxi, and Mount Mufu and Mount Dawei in the Mufu Mountains of northeastern Hunan. This suggests that its geographic distribu-

Table 2. Measurements (in $\mathrm{mm}$ ) of the type series of Rana jiulingensis sp. nov. ( ${ }^{*}=$ holotype).

\begin{tabular}{lcccccccc}
\hline & $\begin{array}{c}\text { SYS } \\
\mathbf{0 0 0 5 5 1 9 *}\end{array}$ & $\begin{array}{c}\text { SYS } \\
\mathbf{a 0 0 5 5 1 1}\end{array}$ & $\begin{array}{c}\text { SYS } \\
\mathbf{a 0 0 6 4 9 4}\end{array}$ & $\begin{array}{c}\text { SYS } \\
\mathbf{0 0 0 6 4 9 5}\end{array}$ & $\begin{array}{c}\text { SYS } \\
\mathbf{0 0 0 2 5 8 4}\end{array}$ & $\begin{array}{c}\text { SYS } \\
\mathbf{a 0 0 2 5 8 5}\end{array}$ & $\begin{array}{c}\text { SYS } \\
\mathbf{0 0 0 6 4 5 1}\end{array}$ & $\begin{array}{c}\text { SYS } \\
\mathbf{0 0 0 6 4 9 6}\end{array}$ \\
\hline Sex & Male & Male & Male & Male & Female & Female & Female & Female \\
SVL & 57.8 & 51.6 & 48.3 & 49.1 & 57.5 & 48.4 & 49.4 & 48.2 \\
HL & 21.6 & 19.3 & 18.4 & 17.7 & 22.3 & 18.9 & 18.2 & 19.4 \\
HW & 18.4 & 17.0 & 15.7 & 12.6 & 19.3 & 16.1 & 15.5 & 15.8 \\
SL & 7.8 & 7.5 & 7.3 & 7.5 & 8.1 & 7.1 & 7.2 & 7.4 \\
IN & 4.1 & 3.8 & 3.5 & 3.2 & 4.2 & 3.6 & 4.1 & 4.1 \\
IO & 3.4 & 2.7 & 3.0 & 3.1 & 3.6 & 3.4 & 3.3 & 3.3 \\
ED & 6.3 & 5.5 & 5.2 & 5.0 & 5.2 & 4.4 & 4.7 & 4.6 \\
TD & 4.6 & 3.5 & 3.3 & 3.9 & 4.5 & 3.7 & 3.2 & 3.4 \\
TED & 1.9 & 2.0 & 1.7 & 2.0 & 1.7 & 1.7 & 1.7 & 1.6 \\
HND & 15.0 & 14.0 & 14.0 & 12.5 & 15.3 & 13.9 & 13.2 & 12.5 \\
RAD & 11.2 & 11.2 & 9.0 & 9.3 & 10.5 & 10.4 & 10.9 & 10.1 \\
FTL & 50.7 & 44.2 & 41.7 & 42.8 & 47.6 & 43.8 & 44.3 & 41.1 \\
TIB & 36.6 & 30.4 & 29.5 & 30.7 & 36.1 & 31.8 & 31.8 & 29.0 \\
\hline
\end{tabular}



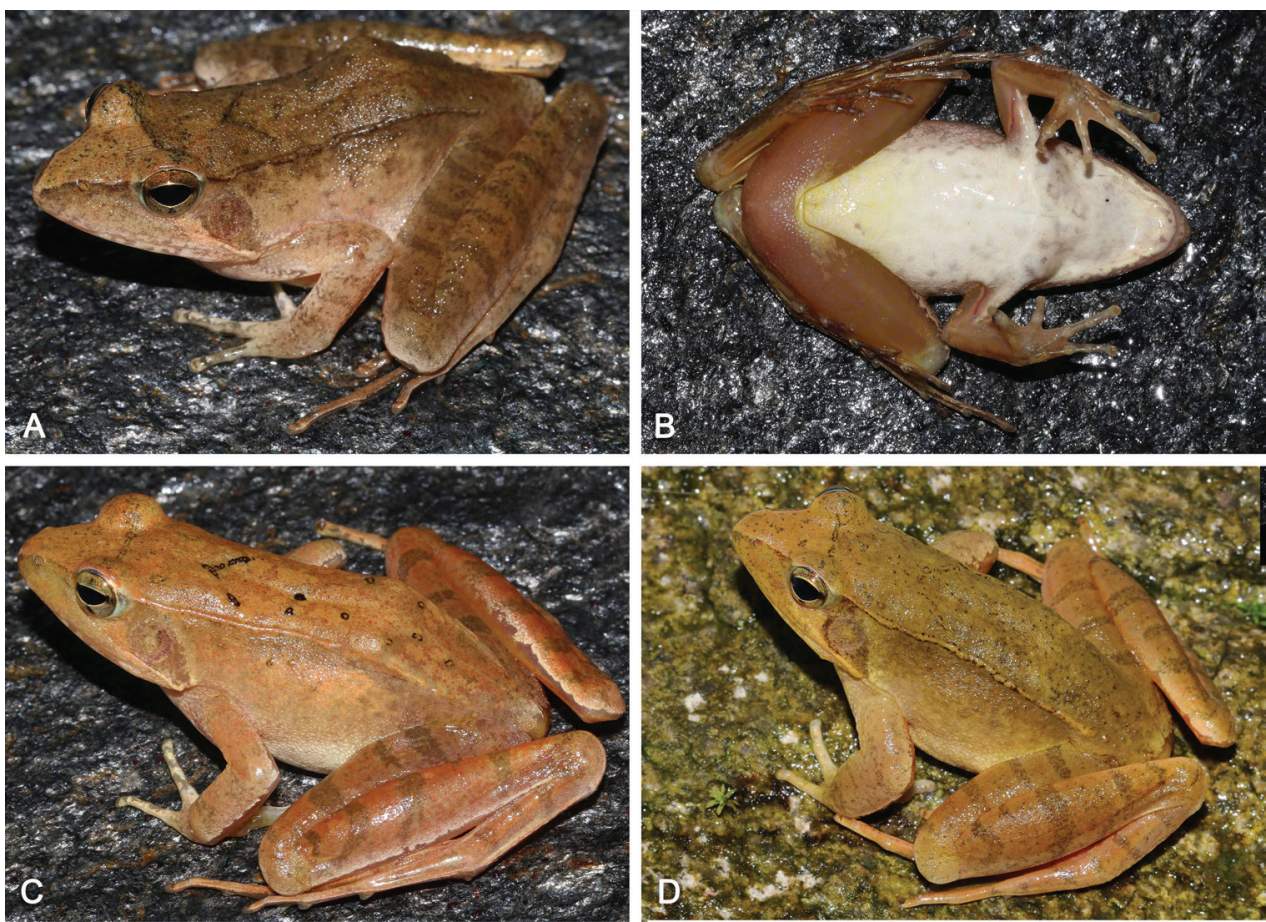

Figure 4. Variations of Rana jiulingensis sp. nov. A, B dorsolateral view and ventral view of male paratype SYS a006495 C male paratype SYS a006496 D male paratype SYS a00511.

tion is the central and northern parts of the Luoxiao Range (Fig. 1). All individuals were found on the surface of paths or on the bush leaves beside paths in subtropical evergreen broadleaved forests. Males SYS a005511 and 5519, which were collected in mid-September, bear a well-developed nuptial pad, while males SYS a006494 and 6495, collected in early August, are without a nuptial pad. This suggests that the breeding season of this species might begin in September.

\section{Discussion}

All recognized species of the subgenus Rana from China (except for $R$. chevronta) are included in our work for morphological and molecular analyses. Four monophyletic clades are supported by high values $(\mathrm{BPP}=1.00$ and $\mathrm{BS}>85$, respectively) in the phylogenetic tree. Three of them correspond to the morphologically recognized $R$. japonica group, $R$. chensinensis group, and $R$. amurensis group. The fourth, unnamed monophyletic clade includes $R$. johnsi, $R$. sangzhiensis, and $R$. zhengi. Within this unnamed clade, $R$. sangzhiensis and $R$. zhengi cluster together with significant support $(\mathrm{BPP}=1.00$ and $\mathrm{BS}=100)$ and little divergence $(0.0-0.4 \%$ in COI and $0.0-0.4 \%$ in $16 S$ ), which is consistent with the original morphological identification by Zheng et al. (1997). Therefore $R$. zhengi is considered a synonym of $R$. sangzhiensis. Furthermore, 
all species of this clade were morphologically previously assigned to Pseudorana (Fei et al. 2009). Thus, based on the phylogenetic relationships and morphological similarities, this monophyletic clade is proposed as a new species group, the Rana johnsi group. For the remaining species, their exact placements remain unresolved due to the insignificant support. Further study of these species is needed, and new species groups might be proposed for these outcast species.

Within the Rana japonica group, the genetic divergences among three species, $R$. longicrus, $R$. zhenhaiensis, and $R$. culaiensis, are relatively closer than other species. Additionally, the validations of these species have been supported by the morphological examinations (Li et al. 2008; Fei et al. 2009). Anuran frogs are suggested with conservative phenotypes (Cherry et al. 1978). Cryptic species, which are morphologically identical but genetically differentiated, are also common in most species complexes (e.g. Yan et al. 2011; Kuraishi et al. 2013; Xiong et al. 2015; Lyu et al. 2019, 2020). With remarkable morphological diversity, but relatively smaller genetic differentiation, $R$. longicrus, $R$. zhenhaiensis, and $R$. culaiensis show a special situation. This suggests that an integrative taxonomic approach is especially important in delimitation of anuran species, and that reliance solely on morphological or molecular evidence would be misleading.

The discovery of Rana jiulingensis sp. nov. increases the diversity of the genus Rana in the Luoxiao Range to five species (Fig. 1). This situation indicates that the Luoxiao Range has the greatest diversity of Rana species in southern China and may be key to speciation of the genus Rana.

\section{Acknowledgements}

We thank the Hunan Badagongshan National Nature Reserve, Fujian Yashushan Nature Reserve, Jian Wang, Chun-Lin Liao, Hai-Long He, Zhao-Chi Zeng, Yu-Long $\mathrm{Li}$, and Zheng-Yan Zhou for their help with the fieldwork. We thank Zhen-Hua Liu, Zi-Chen Zhou, Yang Chen, and Robert Forsyth for their help in polishing the manuscript. We thank Angelica Crottini and two anonymous reviewers for their helpful suggestions on our work. This work was supported by the Project of Comprehensive Scientific Survey of Luoxiao Mountains Region of Ministry of Science and Technology, China (no. 2013FY111500), the Project of Scientific Investigation on the Amphibian, Reptilian and Avian Animals in Jiangxi Jiulianshan National Nature Reserve, the Project of Animal Diversity Survey and Monitoring System Construction of Guangdong Shimentai National Nature Reserve, and the Project of Survey of Terrestrial Vertebrate Diversity in Guangdong Danxiashan National Nature Reserve.

\section{References}

AmphibiaWeb (2019) AmphibiaWeb. University of California, Berkeley, CA, USA. http:// amphibiaweb.org [Accessed on: 2019-9-11] 
Chen X, Chen Z, Jiang J, Qiao L, Lu Y, Zhou K, Zheng G, Zhai X, Liu J (2013) Molecular phylogeny and diversification of the genus Odorrana (Amphibia, Anura, Ranidae) inferred from two mitochondrial genes. Molecular Phylogenetics and Evolution 69: 1196-1202. https://doi.org/10.1016/j.ympev.2013.07.023

Cherry LM, Case SM, Wilson AC (1978) Frog perspective on the morphological difference between humans and chimpanzees. Science 200: 209-211. https://doi.org/10.1126/science.635583

Fei L, Ye CY, Jiang JP (2012) Colored Atlas of Chinese Amphibians and their Distributions. Sichuan Publishing House of Science and Technology, Chengdu. [In Chinese]

Fei L, Hu SQ, Ye CY, Huang YZ (2009) Fauna Sinica. Amphibia Vol. 2 Anura. Science Press, Beijing. [In Chinese]

Frost DR (2020) Amphibian Species of the World: an Online Reference. Version 6.0. American Museum of Natural History, New York, USA. http://research.amnh.org/herpetology/ amphibia/index.html [Accessed on: 2020-4-29]

Hu SQ, Fei L, Ye CY (1978) Three new amphibian species in China. Materials for Herpetological Research 4: 20.

Kuraishi N, Matsui M, Hamidy A, Belabut DM, Ahmad N, Panha S, Sudin A, Yong HS, Jiang JP, Ota H, Thong HT, Nishikawa K (2013) Phylogenetic and taxonomic relationships of the Polypedates leucomystax complex (Amphibia). Zoologica Scripta 42: 54-70. https://doi. org/10.1111/j.1463-6409.2012.00562.x

Li PP, Lu YY, Li A (2008) A new species of brown frog from Bohai, China. Asiatic Herpetological Research 11: 60-68.

Liu CC (1946) A new woodfrog Rana chaochiaoensis with a discussion of its allied species, from West China. Journal of the West China Border Research Society, Series B 16: 7-14.

Liu MY, Zhang SQ, Liu M (1993) A new species of Ranidae from Liaoning, China (Anura). Acta Zootaxonomica Sinica 18: 493-497.

Lu YY, Li PP, Jiang DB (2007) A new species of Rana (Anura, Ranidae) from China. Acta Zoologica Sinica 32: 792-801.

Lyu ZT, Huang LS, Wang J, Li YQ, Chen HH, Qi S, Wang YY (2019) Description of two cryptic species of the Amolops ricketti group (Anura, Ranidae) from southeastern China. ZooKeys 812: 133-156. https://doi.org/10.3897/zookeys.812.29956

Lyu ZT, Dai KY, Li Y, Wan H, Liu ZY, Qi S, Lin SM, Wang J, Li YL, Zeng YJ, Li PP, Pang H, Wang YY (2020) Comprehensive approaches reveal three cryptic species of genus Nidirana (Anura, Ranidae) from China. ZooKeys 914: 127-159. https://doi.org/10.3897/zookeys.914.36604

Meyer CP, Geller JB, Paulay G (2005) Fine scale endemism on coral reefs: archipelagic differentiation in turbinid gastropods. Evolution 59: 113-125. https://doi. org/10.1111/j.0014-3820.2005.tb00899.x

Ronquist F, Teslenko M, Van Der Mark P, Ayres DL, Darling A, Höhna S, Larget B, Liu L, Suchard MA, Huelsenbeck JP (2012) MrBayes 3.2: efficient Bayesian phylogenetic inference and model choice across a large model space. Systematic Biology 61: 539-542. https://doi.org/10.1093/sysbio/sys029

Savage JM (1975) Systematics and distribution of the Mexican and Central American stream frogs related to Eleutherodactylus rugulosus. Copeia 2: 254-306. https://doi. org/10.2307/1442883 
Shen Y, Jiang J, Yang D (2007) A new species of the genus Rana-Rana hanluica sp. nov. from Hunan Province, China (Anura: Ranidae). Acta Zoologica Sinica 53: 481-488.

Simon C, Frati F, Beckenbach A, Crespi B, Liu H, Flook P (1994) Evolution, weighting, and phylogenetic utility of mitochondrial gene sequences and a compilation of conserved polymerase chain reaction primers. Annals of the Entomological Society of America 87: 651701. https://doi.org/10.1093/aesa/87.6.651

Silvestro D, Michalak I (2012) RaxmlGUI: a graphical front-end for RAxML. Organisms Diversity and Evolution 12: 335-337. https://doi.org/10.1007/s13127-011-0056-0

Stejneger L (1898) On a collection of batrachians and reptiles from Formosa \& adjacent islands. Journal of the College of Science 12: 215-225.

Tamura K, Stecher G, Peterson D, Filipski A, Kumar S (2013) MEGA6: molecular evolutionary genetics analysis, version 6.0. Molecular Biology and Evolution 30: 2725-2729. https://doi.org/10.1093/molbev/mst197

Thompson JD, Gibson TJ, Plewniak F, Jeanmougin F, Higgins DG (1997) The CLUSTAL_X windows interface: flexible strategies for multiple sequence alignment aided by quality analysis tools. Nucleic Acids Research 25: 4876-4882. https://doi.org/10.1093/ nar/25.24.4876

Wang C, Qian L, Zhang C, Guo W, Pan T, Wu J, Wang H, Zhang B (2017) A new species of Rana from the Dabie Mountains in eastern China (Anura, Ranidae). ZooKeys 724: 135-153. https://doi.org/10.3897/zookeys.724.19383

Wang JQ, Li PP, Lu YY, Dong BJ, Zhou ZY, Yu FL (2006) Description and comparison of tadpoles of Rana huanrenensis and $R$. dybowskii. Sichuan Journal of Zoology 45: 349-353. [In Chinese]

Xiong R, Li C, Jiang J (2015) Lineage divergence in Odorrana graminea complex (Anura: Ranidae: Odorrana). Zootaxa 3963: 201-229. https://doi.org/10.11646/zootaxa.3963.2.3

Yan F, Jiang K, Chen H, Fang P, Jin J, Li Y, Wang S, Murphy RW, Che J, Zhang Y (2011) Matrilineal history of the Rana longicrus species group (Rana, Ranidae, Anura) and the description of a new species from Hunan, southern China. Asian Herpetological Research 2: 61-71. https://doi.org/10.3724/SP.J.1245.2011.00061

Yang BT, Zhou Y, Min MS, Matsui M, Dong BJ, Li PP, Fong JJ (2017) Diversity and phylogeography of Northeast Asian brown frogs allied to Rana dybowskii (Anura, Ranidae). Molecular Phylogenetics and Evolution 112: 148-157. https://doi.org/10.1016/j. ympev.2017.04.026

Ye CY, Fei L, Hu SQ (1993) Rare and economic amphibians of China. Sichuan Publishing House of Science and Technology, Chengdu. [In Chinese]

Ye CY, Fei L, Matsui M (1995) Taxonomic studies of Chinese Rana japonica Guenther. Acta Herpetologica Sinica 4: 82-87.

Yuan ZY, Zhou WW, Chen X, Poyarkov Jr. NA, Chen H-M, Jang-Liaw N-H, Chou WH, Matzke NJ, Iizuka K, Min M-S, Kuzmin SL, Zhang YP, Cannatella DC, Hillis DM, Che J (2016) Spatiotemporal diversification of the true frogs (genus Rana): a historical framework for a widely studied group of model organisms. Systematic Biology 65: 824-842. https://doi.org/10.1093/sysbio/syw055 
Zhao H, Yang J, Wang C, Li P, Yuan Z (2017) A new species of the genus Rana from Henan, central China (Anura,Ranidae). ZooKeys 694: 95-108. https://doi.org/10.3897/zookeys.694.12513

Zheng MQ, Fei L, Jiang JP, Xie F (1997) A preliminary research on the relations between ecological environment and the status of herpetological resources in Wawushan Mountain National Forest Park. Cultum Herpetologica Sinica 6-7: 67-74.

Zhou Y, Wang SR, Zhu HD, Li PP, Yang BT, Ma JZ (2017) Phylogeny and biogeography of south Chinese brown frogs (Ranidae, Anura). PloS ONE 12: e0175113 https://doi. org/10.1371/journal.pone.0175113

Zhou Y, Yang BT, Li PP, Min MS, Fong JJ, Dong BJ, Zhou ZY, Lu YY (2015) Molecular and morphological evidence for Rana kunyuensis as a junior synonym of Rana coreana (Anura: Ranidae). Journal of Herpetology 49: 302-307. https://doi.org/10.1670/13-111

\section{Appendix I}

\section{Specimens examined}

Rana chaochiaoensis (3): China: Sichuan: Zhaojue County (type locality): SYS a001815-1816, 1831.

Rana chensinensis (2): China: Henan: Mt Yawu: SYS a002392-2393.

Rana culaiensis (4): China: Jiangxi: Mt wugong: SYS a002634; Shanggao County: SYS a002641;Mt Meiling: SYS a004239,4241.

Rana hanluica (35): China: Hunan: Mt Yangming (type locality): SYS a0011371147; Mt Bamian: SYS a004086; Mt Badagong: SYS a004298; Mt Yunshan: SYS a004358-4359; Mt Xuefeng: SYS a007216; Suining County: SYS a007250-7251; Mt Shunhuang: SYS a007259-7260; Guizhou: Mt Leigong: SYS a002233; Mt Fanjing: SYS a004346; Jiangxi: Mt Jinggang: SYS a004195-4196; Mt Qiyun: SYS a004087; Guangxi: Longsheng County: SYS a002284-2288; Mt Dupangling: SYS a005086-5088; Guangdong: Renhua County: SYS a007009-7100.

Rana jiemuxiensis (2): China: Hunan: Jiemuxi Nature Reserve (tpye locality): SYS a004318-4319.

Rana kukunoris (7): China: Sichuan: Hongyuan County: SYS a006652-6654; Maoxian County: SYS a005381-5384.

Rana longicrus (18): China: Fujian: Mt Yashu: SYS a005892, 5905; Guangdong: Renhua County: SYS a000732-0735, 5624-5625; Mt Nankun: SYS a000754, 4589, 5579; Yingde City: SYS a00 7519; Pu'ning City: SYS a004605; Mt Tonggu: SYS a005808; Jiangxi: Mt Qiyun: SYS a002355; Mt Jiulian: SYS a004487; Mt Magu: SYS a007038; Suichuan County: SYS a007097.

Rana omeimontis (5): China: Sichuan: Mt Emei (type locality): SYS a005304-5305; Anzhou District: SYS a005393; Guizhou: Qixingguan District: SYS a007294-7295.

Rana zhenhaiensis (7): China: Zhejiang: Fenghua District: SYS a006208, 7506-7507; Jiangxi: Mt Tongbo: SYS a001951-1953; Guanshan Nature Reserve: SYS a007000. 


\section{Supplementary material I}

Tables S1, S2

Authors: Han Wan, Zhi-Tong Lyu, Shuo Qi, Jian Zhao, Pi-Peng Li, Ying-Yong Wang

Data type: Tables for genetic distances

Explanation note: Table S1. Pairwise distances based on COI gene among all sample used in this study. Table S2. Pairwise distances based on 16 S gene among all sample used in this study.

Copyright notice: This dataset is made available under the Open Database License (http://opendatacommons.org/licenses/odbl/1.0/). The Open Database License $(\mathrm{ODbL})$ is a license agreement intended to allow users to freely share, modify, and use this Dataset while maintaining this same freedom for others, provided that the original source and author(s) are credited.

Link: https://doi.org/10.3897/zookeys.942.46928.suppl1 